\title{
Evaluation of three commercial enzyme immunoassay kits for detecting faecal Clostridium difficile toxins
}

\author{
S A Arrow, L Croese, R A Bowman, T V Riley
}

\begin{abstract}
The detection of faecal cytotoxicity using tissue culture was compared with three commercial Clostridium difficile enzyme immunoassay (EIA) kits; Premier C difficile toxin A (Meridian Diagnostic, Inc.); CD-TOX $C$ difficile toxin A (Porton Cambridge); and Cytoclone A + B EIA (Cambridge Biotech Corporation). Of 160 faecal samples examined by all four methods, 52 (32.5\%) were cytotoxic, 44 (27.5\%) were positive by Premier, 48 (30\%) by CD-TOX EIA, and 50 (31.3\%) with Cytoclone. When compared with detection of cytotoxicity by tissue culture assay, the following performance indices were obtained: Premier, sensitivity $84 \cdot 1 \%$, specificity $99 \cdot 1 \%$, positive predictive value (PPV) 97.8\%, negative predictive value (NPV) 93\%; CD-TOX, sensitivity $92 \cdot 3 \%$, specificity $88 \cdot 0 \%$, PPV 78.7\%, NPV 95.9\%; Cytoclone, sensitivity 96.2\%, specificity $93.5 \%$, PPV $87 \cdot 7 \%$, NPV 98.1\%. EIA results were available within three hours, whereas the results of the cytotoxin assay were available after 24-48 hours. All three kits provided satisfactory results and, although relatively expensive, all could be used in the laboratory effectively to screen for diarrhoeal disease associated with $\boldsymbol{C}$ difficile.
\end{abstract}

(F Clin Pathol 1994;47:954-956)

Clostridium difficile is the major cause of pseudomembranous colitis and antibiotic associated diarrhoea. ${ }^{1}$ Culture of the organism or demonstration of cytotoxicity remain the most commonly used tests for the laboratory diagnosis of $C$ difficile associated diarrhoea. Culture of the organism is sensitive, although time consuming, but lacks specificity as nontoxigenic strains will also be detected. ${ }^{2}$ Detection of cytotoxicity using a tissue culture assay has been used as the "gold standard" for the diagnosis of $C$ difficile associated diarrhoea because of its high sensitivity and specificity. However, many smaller laboratories are not in a position to undertake tissue culture techniques due to the lack of experience or equipment to perform the test. Recently developed commercial enzyme immunoassay (EIA) kits are now available to detect the presence of toxins A or B. ${ }^{3-6}$ These kits base the detection of toxin A or B on the use of specific antibody bound to a solid phase (microtitre well). We report here an evaluation of three EIA kits for the detection of $C$ difficile toxins in faecal samples.

\section{Methods}

Faecal specimens were collected between January and July 1993, from patients in Sir Charles Gairdner Hospital (SCGH), a 680 bed general teaching hospital. Specimens referred to SCGH from other hospitals and laboratories were also included. After routine culture for $C$ difficile and testing for cytotoxin in tissue culture, the specimens were frozen at $-20^{\circ} \mathrm{C}$ and later tested in batches with the three commercial kits. Tissue culture assays were also repeated to assess any degradation of the toxin(s) following the freeze/thaw cycle.

Vero cells were used to detect $C$ difficile cytotoxicity. Cells were grown in Eagle's minimal essential medium supplemented with $3 \%$ fetal calf serum, $10 \mathrm{mg} / \mathrm{l}$ of gentamicin, and $10 \mathrm{mg} / 1$ of vancomycin. Cell lines were grown in an atmostphere of $10 \% \mathrm{CO}_{2}$ at $37^{\circ} \mathrm{C}$. Faecal filtrates were prepared for detection of cytotoxicity by emulsifying about $1 \mathrm{~g}$ of faeces in $3 \mathrm{ml}$ of saline and centrifuging at $2500 \mathrm{rpm}$ for five minutes. After filtering through a 0.45 $\mu \mathrm{m}$ Millipore filter, $100 \mu \mathrm{l}$ of filtrate was added to two wells of a multiwell $(16 \mathrm{~mm})$ Costar tissue culture plate seeded with Vero cells. To one well $100 \mu \mathrm{l}$ of Clostridium sordellii antitoxin (Wellcome) was added. After 48 hours of incubation, the cells were examined for typical changes (rounding). Wells exhibiting over $80 \%$ rounding were considered positive.

Direct and enrichment culture for $C$ difficile was performed by the methods described before. $^{7}$

Kits used in the study were: (i) Premier $C$ difficile Toxin A (Meridian Diagnostic, Inc.); (ii) CD-TOX $C$ difficile Toxin A (Porton Cambridge); (iii) Cytoclone Toxin A + B EIA (Cambridge Biotech Corporation). All kits were used according to the manufacturer's instructions. All kits recommended the preparation of a $20 \%$ faecal suspension in the relevant diluent. All results were read by eye on a white background and colour intensity was compared with that of the negative control.

\section{Results}

One hundred and sixty faecal specimens were cultured for $C$ difficile and tested by tissue culture assay and the three commercial kits. Fifty two $(32.5 \%)$ were positive by tissue culture assay when tested after freeze/thawing. Seven 
Performance indices of ELA kits compared with tissue culture assay

\begin{tabular}{llll}
\hline & Premier & CD-TOX & Cytoclone $A+B$ \\
\hline Sensitivity & $\mathbf{8 4 . 6 \%}$ & $92 \cdot 3 \%$ & $96 \cdot 2 \%$ \\
Specificity & $99 \cdot 1 \%$ & $88.0 \%$ & $93.5 \%$ \\
PPV & $97 \cdot 8 \%$ & $78 \cdot 7 \%$ & $87 \cdot 7 \%$ \\
NPV & $93.0 \%$ & $95.9 \%$ & $98 \cdot 1 \%$ \\
\hline
\end{tabular}

samples were initially positive, but negative after freeze/thawing. Seven specimens were positive by culture but negative with all the kits and on cytotoxicity testing. Two of these specimens yielded cytotoxigenic strains of $C$ difficile on culture and in vitro testing. The remaining five isolates were non-cytotoxigenic.

Of the 52 specimens positive on repeat cytotoxicity testing, 44 were positive with the Premier kit. Of the remaining 108 specimens negative by tissue culture assay, 107 were negative with the Premier kit. The one Premier positive, cytotoxicity negative specimen was culture negative but positive with the Cytoclone. All specimens negative by Premier kit were negative for $C$ difficile by culture.

Of the $\mathbf{5 2}$ specimens positive by tissue culture, 48 were positive with the CD-TOX kit. Of the 108 specimens negative by tissue culture, 95 were negative with CD-TOX. Of the 13 CD-TOX positive specimens but negative for cytotoxicity, five yielded a cytotoxigenic strain of $C$ difficile by culture and subsequent in vitro testing.

Fifty of the 52 specimens positive by tissue culture were positive with the Cytoclone kit. Of the 108 specimens negative by tissue culture, 101 were negative with the Cytoclone kit. Two of these specimens yielded cytotoxigenic strains of $C$ difficile.

The sensitivity, specificity, positive predictive value (PPV) and negative predictive value (NPV) of the three commercial kits compared with the tissue culture assay are shown in the table.

\section{Discussion}

Traditionally, the detection of $C$ difficile toxin in the laboratory has been performed by tissue culture assay because of its extreme sensitivity and specificity. However, many small laboratories do not have either the experience or the facilities to perform tissue culture techniques. The incubation period for detection of cytotoxin in tissue culture may also be up to 48 hours. A toxin detection method which does not use tissue culture and which needs less incubation time would be ideal.

Rapid EIA techniques for the detection of $C$ difficile toxin(s) are now commercially available. This study evaluated three: Premier $C$ difficile toxin A (Meridian Diagnostic, Inc.); CD-TOX $C$ difficile toxin A (Porton Cambridge); and Cytoclone $C$ difficile $\mathrm{A}+\mathrm{B}$ EIA (Cambridge Biotech Corporation). They were compared with our reference method for cytotoxin detection, a tissue culture assay using Vero cells. Overall, the kits performed exceptionally well with very little difference between kits. The Premier kit was the most specific $(99 \cdot 1 \%)$, with a sensitivity of $84.6 \%$ and the Cytoclone was the most sensitive $(96 \cdot 2 \%)$, with a specificity of $93 \cdot 5 \%$.

With respect to the CD-TOX results, of 13 specimens negative by tissue culture but positive by CD-TOX, five yielded cytotoxigenic strains of $C$ difficile. With this taken into consideration, the specificity of the CD-TOX kit increased from $88.0 \%$ to $92 \cdot 6 \%$. Similar results were obtained with Cytoclone. In this case, of seven specimens negative by tissue culture but positive by Cytoclone, two were culture positive for cytotoxigenic strains of $C$ difficile. This improved the specificity of the Cytoclone from $93.5 \%$ to $95 \cdot 4 \%$. After taking these changes into consideration, the kit with the best performance indices was the Cytoclone with a sensitivity of $96.2 \%$ and a specificity of $93.5 \%$.

It is interesting to speculate as to the reasons why some specimens were negative by tissue culture but positive by EIA methods. Seven culture positive specimens which were initially positive by tissue culture but negative after freeze/thawing were EIA (toxin A and B) positive. This suggests that the freeze/thawing cycle decreased the biological activity, but not the immunological activity of the toxin(s).

Several studies have evaluated various EIA kits for the detection of $C$ difficile toxin $A$ and toxin B since they became available..$^{3-6}$ Several of these have assessed the Premier kit. Di Persio et $a l^{8}$ compared the Premier kit with a stool cytotoxicity assay and achieved figures for sensitivity and specificity of 84.1 and $98.9 \%$, respectively. In a multicentre study of 504 samples, the sensitivity and specificity of the Premier kit were 86.6 and $99.0 \%$, respectively, while the PPV and NPV were 94.7 and $97 \cdot 4 \%$, respectively. ${ }^{6}$ Borriello et al ${ }^{3}$ evaluated the Premier kit with 101 specimens of which 69 were positive by isolation of organism or cytotoxicity assay. Forty nine of these 69 were positive using the Premier kit. $C$ difficile was cultured from $25 \%$ of specimens negative by cytotoxin or Premier assay, possibly suggesting a lack of sensitivity of the kit. Finally, Delmee $e t a l^{4}$ determined the sensitivity and specificity of the Premier EIA kit to be 88 and $95 \%$, respectively, when compared with tissue culture using Hela cells in a study of 228 specimens. Our results are similar to those quoted above, with good specificity $(99 \cdot 1 \%)$ and reasonable sensitivity (84.6\%).

Fewer investigators have evaluated the Cytoclone A + B EIA kit. This test has the potential advantage of being able to detect both toxin A and toxin B. Most strains of $C$ difficile produce both toxins, but the relative amounts of toxin produced may vary between strains. Doern et $a l^{5}$ compared this test with a cytotoxicity assay and recorded both a high sensitivity (94.9\%) and specificity (98.9\%). While we found a slightly higher sensitivity $(96 \cdot 2 \%)$, our specificity was slightly lower $(93.5 \%)$. The sensitivity recorded was the best of the three kits evaluated and may be a reflection of the Cytoclone A + B kit's ability to detect both toxins. In addition, a high NPV 
(98.1\%) was calculated, suggesting that the Cytoclone A + B kit would make a useful screening test.

To our knowledge the CD-TOX kit has not been evaluated before. Although the figures for specificity and PPV were not as high as those for the other two kits, the high NPV (96.0\%) would also make the CD-TOX a good screening test.

Procedures for using the Premier and the CD-TOX kits were similar. With the Premier kit, results were available in about two hours 15 minutes, while with the CD-TOX, results take only about one hour 15 minutes. The Cytoclone kit was slightly more time consuming involving a centrifugation step and also more wash steps than the other kits, and results were not available for about three hours.

Both the Premier and the Cytoclone kits cost about $\$ 9.40$ (roughly $£ 4.60$ ) per specimen while the CD-TOX kit cost is about $\$ 6.25$ (roughly $£ 3.00$ ) per specimen. These costs do not allow for controls and it should be noted that each batch of specimens must have at least one positive and one negative control. The CD-TOX kit actually recommends the use of four controls with each batch, thus increasing the relative cost per specimen. It is difficult to estimate costs of tissue culture techniques as there are many hidden factors requiring consideration. The laboratory must also have appropriate facilities to maintain the cell lines and have staff familiar with these techniques.

In conclusion, small laboratories without facilities for tissue culture should find the EIA kits an excellent alternative, with all three kits performing well. An automatic plate washer and reader would further enhance the efficiency of these techniques. The CD-TOX kit is the least expensive, but if the recommendations of the manufacturer are followed, and four controls per batch are used, the cost per specimen is substantially increased. The Cytoclone kit gave the most impressive performance indices, but the test takes slightly longer to complete. All kits can be recommended and it must be left to individual laboratories to decide whether tissue culture methods or EIA kits meet the logistic needs or financial requirements of their laboratory.

1 Lyerly DM, Krivan HC, Wilkins TD. Clostridium difficile: its disease and toxins. Clin Microbiol Rev 1988;1:1-18.

2 Knoop FC, Ownes MA, Crocker IC. Clostridium difficile: Clinical disease and diagnosis. Clin Microbiol Rev 1993; 6:251-65.

3 Borriello SP, Vale T, Brazier JS, Hyde S, Chippick E. Evaluation of a commercial kit for the detection of Clostridium difficile toxin A. Eur $f$ Clin Microbiol Infect Dis 1992tridium difficile

4 Delmee M, Mackey T, Hamitou A. Evaluation of a new commercial Clostridium difficile toxin A enzyme immunoassay using diarhoeal stools. Eur $\mathcal{f}$ Clin Microbiol Infect Dis 1992;11:246-9.

5 Doern GV, Coughlin RT, Wu L. Laboratory diagnosis of Clostridium difficile-associated gastrointestinal disease: comparison of a monoclonal antibody enzyme immunocor for toxins A and B with a monoclonal antibody a monoclonal antibody city assays. 7 Clin Microbiol 1992;30:2042-6.

city assays. F Clin Microbiol 1992;30:2042-6.
De Girolami PC, Hanff PA, Eichelberger K, Longhi L, Teresa H, Pratt J, et al. Multicenter evaluation of a new Teresa $\mathrm{H}$, Pratt J, et al. Multicenter evaluation of a new enzyme immunoassay for detection of Clostridiu

7 Bowman RA, Riley TV. The laboratory diagnosis of $C$ dif ficile-associated diarrhoea. Eur $\mathcal{f}$ Clin Microbiol Infect Dis 1988;7:476-84.

8 Di Persio JR, Varga FJ, Conwell DL, Kraft JA, Kozak KJ, Willis DH. Development of a rapid enzyme immunoassay for Clostridium difficile toxin $\mathrm{A}$ and its use in the diaonosis of $C$ difficile-associated disease. $\mathcal{F}$ Clin Microbiol 1991;29. 2724-30.

\title{
Imerslund-Grasbeck syndrome in a Chinese family with distinct skin lesions refractory to vitamin $B_{12}$
}

\author{
S-H Lin, N A Sourial, K-C Lu, E-J Hsueh
}

\author{
Division of \\ Haematology and \\ Oncology, \\ Department of \\ Medicine, Tri-Service \\ General Hospital, \\ National Defense \\ Medical Centre, \\ 8 Ting-Chow Road, \\ Number 8, Section 3 , \\ Taipei, Taiwan \\ S-H Lin \\ K-C Lu \\ E-J Hsueh \\ Department of \\ Haematology, St \\ Bartholomew's \\ Hospital, London \\ N A Sourial \\ Correspondence to: \\ Dr Shih-Hua Lin \\ Accepted for publication \\ 24 March 1994
}

\begin{abstract}
Two brothers in a Chinese family with selective malabsorption of vitamin $B_{12}$ associated with proteinuria (ImerslundGrasbeck syndrome) presented with widespread mottled skin pigmentation, termed poikiloderma. In contrast to anaemia, this pigmentary disturbance remained unresponsive to vitamin $B_{12}$ replacement. This is different from the reported hyperpigmentation sometimes seen in vitamin $B_{12}$ deficiency which is reversible following treatment. As far as is known, an irreversible and persistent skin disorder has not been reported in this syndrome before.
\end{abstract}

\section{(F Clin Pathol 1994;47:956-958)}

Familial selective malabsorption of vitamin $B_{12}$ associated with proteinuria was first described by Imerslund (1960) ${ }^{1}$ and Grasbeck et al $(1960)^{2}$ (Imerslund-Grasbeck syndrome). It is inherited as an autosomal recessive trait and is prevalent among small inbred communities such as the Lapps in Scandinavia and North African Jews. ${ }^{3}$ Only a few cases had been reported in Orientals. ${ }^{4} \mathrm{We}$ have seen two young men in a Chinese family with this syndrome associated with a skin disorder, known as poikiloderma. The anaemia in both patients responded to vitamin $B_{12}$ treatment but the skin lesions did 